\title{
Relook on fitting of viscosity with undercooling of glassy liquids
}

\author{
C CHATTOPADHYAY, S SANGAL and K MONDAL* \\ Department of Materials Science and Engineering, Indian Institute of Technology, Kanpur 208 016, India
}

MS received 9 September 2012; revised 2 October 2013

\begin{abstract}
The present approach is on the modification of viscosity fitting of undercooled liquid as a function of undercooling. The method consists of finding analytical solution of three arbitrary constants of the Vogel-FulcherTamman (VFT) equation by choosing three viscosity data at three critical temperatures for an undercooled liquid. Three critical temperatures are liquidus temperature $\left(T_{1}\right)$, crystallization onset temperature $\left(T_{\mathrm{x}}\right)$ and glass transition temperature $\left(T_{\mathrm{g}}\right)$. The experimental viscosity data at or very near to these three critical temperatures (depending on the availability in literature) have been utilized to achieve the analytical solution. The analytical solution of VFT equation is further examined by selecting the experimental data points away from the critical temperatures in order to check their $\left(T_{\mathrm{l}}, T_{\mathrm{x}}\right.$ and $\left.T_{\mathrm{g}}\right)$ significance towards the solution. Total absolute error (TAE) and total squared error (TSE) values obtained from the present method with respect to the experimental viscosity data in the temperature range between $T_{1}$ and $T_{\mathrm{g}}$ are very much comparable and in most of the cases lower than that of existing 'best-fit' cited in the literature for a number of glassy alloys. Moreover, this method interestingly enables us to find the fragility parameters for a number of glassy alloys and convincingly explain their true glass forming abilities (GFA).
\end{abstract}

Keywords. Viscosity; fragility; bulk metallic glass; glass forming ability.

\section{Introduction}

Viscosity of a liquid is one of the key kinetic parameters for understanding liquid-solid phase transformations like nucleation and growth of solid in liquid, time-temperaturetransformation diagram, glass forming ability for undercooled liquids, etc. Viscosity is a function of temperature and Doolittle (1951) proposed a very simple approach to determine viscosity as a function of temperature where viscosity is assumed to be dependent on relative free space. Cohen and Grest (1979) proposed an empirical relationship between viscosity and temperature where they have correlated viscosity with free volume and thereby, the relaxation. It is shown that the relationship holds good for a number of glassy compounds and many inorganic as well as organic compounds. However, the relationship is associated with five arbitrary constants and becomes complex. Battezzati and Greer (1989) studied viscosity of elements and alloys at widely varying temperatures and tried to evaluate a temperature dependence of viscosity. They have shown that Vogel-FulcherTamman (VFT) equation describes the temperature dependence of viscosity in the best way for temperatures below melting point to glass transition temperature. Angel (1988) has explained that the viscosity-temperature relationship can be well described by power law expression, Arrhenius expression and VFT equation. However, the abrupt change of viscosity (viscosity divergence) towards the temperature, $T_{\mathrm{x}}$, can be explained satisfactorily by VFT expression. Furthermore, Angel (1995) extensively showed that VFT equation

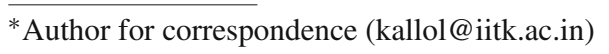

shows perfect behaviour for glassy alloys. Thus, VFT equation is chosen amongst all as the best description of viscosity behaviour of glassy alloys and organic and ionic compounds in the temperature range between $T_{1}$ and $T_{\mathrm{g}}$. The viscosity, $\eta$, at any temperature, $T(\mathrm{~K})$, relates to temperature as per VFT equation

$$
\eta=\eta_{0} \exp \left(\frac{D T_{0}}{T-T_{0}}\right),
$$

where $\eta_{0}, D, T_{0}$ are pre-exponential constant, fragility parameter of the glassy material and ideal glass transition temperature, respectively. VFT equation has been treated in the same way as per the work described by Angel (1995). Angel has considered $D$ to be the parameter which decides how closely the system obeys Arrhenius law ( $D=$ infinite). Like many others, $D$ has also been considered as fragility parameter by Busch (2000) and it is related to the strength of a liquid or how strong is the liquid. However, it is to be made clear that 'fragility parameter' does not mean 'fragility' (F) of a liquid. In this context, various definitions of fragility of glassy materials expressed by several researchers have been vividly explained in the work of Rao et al (2001). As per Angel (1986), fragility $(F)$ of a liquid can be the inverse of $D$. The fragility parameter $D$ is very essential for judging glass forming ability of a glass. Angel (1995) described glasses on the basis of the values of $D$ of VFT expression for a number of glassy materials as strong or fragile. A number of research works have been carried out on the evaluation of temperature dependence of viscosity on the basis of this equation (Laughlin and Uhlmann 1972; Polk and Turnbull 1972; Waniuk et al 1998; Masuhr et al 1999; Mukherjee et al 2004; 
Ojovan 2004; Nascimento and Aparicio 2007; Avramov 2011). The method so far used is to get experimental viscosity data of a liquid alloy at various temperatures and then fit a curve according to (1) to achieve values of the three VFT parameters $\left(\eta_{0}, D\right.$ and $\left.T_{0}\right)$. Glassy alloys enable to determine viscosity of liquid in the temperature range between $T_{1}$ and $T_{\mathrm{g}}$ because of its inherent stability against crystallization (Masuhr et al 1999). However, experimental measurement of liquid viscosity could also be decided by the strong and weak nature of the liquid. Hence, up to certain level of undercooling below melting temperature, viscosity can be measured and again another temperature range where experimental viscosity can be obtained is between crystallization onset temperature and glass transition temperature. Hence, for achieving a viscosity value in between these two ranges, interpolation is inevitable. The interpolation invariably depends on the goodness of fit of the function which undoubtedly depends on number of experimental data points available for any fitting technique (Mukherjee et al 2004). Therefore, it is expected that more are the number of data points, less erroneous will be the interpolation. However, there exists a finite error in each and every data point for any fitting. The three parameters estimated from VFT equation through the mentioned way have great significance with respect to the real behaviour of the glassy alloys. The fragility parameter, $D$, for example, is a measure of how fragile the liquid is and accordingly, its glass forming ability can be decided. However, better the fit is, better will be the value of all the parameters including $D$. One possible approach could be finding three known viscosities at three temperatures and from that an analytical solution of (1) can be possible. Hong et al (1996) have shown similar kind of method where they have taken the viscosity data in the form of activation energy at three temperatures and the calculated values of three parameters in VFT equation show large variation in all the three methods used in their study. The currently proposed method is based on analytical solution approach from the three viscosity values at three critical temperatures $\left(T_{1}, T_{\mathrm{x}}\right.$ and $\left.T_{\mathrm{g}}\right)$. TAE and TSE as calculated with respect to the experimental data in the entire range of viscosities between $T_{1}$ and $T_{\mathrm{g}}$, for the present method have been compared to the corresponding best-fit available in literature. Furthermore, the $D$ values as calculated in the proposed method interestingly enable us to understand the true glass forming abilities (GFA) of a number of glassy alloys.

\section{Theory}

If three data sets of viscosity and temperature are available i.e. $\left(\eta_{1}, T_{1}\right)$ (data set 1$),\left(\eta_{2}, T_{2}\right)$ (data set 2$)$ and $\left(\eta_{3}, T_{3}\right)$ (data set 3 ), each of them will satisfy (1). Thus, by putting three data sets and taking natural logarithm on both sides, (1) becomes

$$
\ln \eta_{1}=\ln \eta_{0}+\frac{D T_{0}}{T_{1}-T_{0}},
$$

$$
\begin{aligned}
& \ln \eta_{2}=\ln \eta_{0}+\frac{D T_{0}}{T_{2}-T_{0}}, \\
& \ln \eta_{3}=\ln \eta_{0}+\frac{D T_{0}}{T_{3}-T_{0}} .
\end{aligned}
$$

Solving (2), (3) and (4), three constants in VFT equation can be obtained. Mathematically, any three temperatures with their respective viscosity values can be chosen to get an analytical solution of (1). However, a set of three points results in a solution which does not exactly pass through all the other experimental data points. How close the solution will pass through the other points can be estimated by quantifying total errors for rest of all the points. Hence, the choice of data points should be in such a fashion which minimizes the total error. For the present work, the temperature range is fixed to be $T_{1}$ to $T_{\mathrm{g}}$ as stated earlier. At melting temperature, the atomic movement becomes much easier, thereby, giving rise to less relaxation time. In that respect, $T_{1}$ is a 'junction' point. At glass transition temperature, $T_{\mathrm{g}}$, the atoms totally freeze giving rise to difficulty in shear movement of atoms and it results in increase of the relaxation time. The viscosity jumps to a very high value at this temperature. Therefore, it is also taken as a 'junction temperature'. Now, the third point must lie in between $T_{1}$ and $T_{\mathrm{g}}$ and this is the most important selection. If the viscosity behaviour in this range is carefully observed, one more 'junction temperature' could be found out. Scudino et al (2009) have clearly shown that near $T_{\mathrm{x}}$ temperature, the viscosity of glassy alloys increases abruptly because of losing liquid like behaviour. They have further proposed that before crystallization the alloy converts itself to a quasicrystalline state and this has been described as relaxed iso-configurational state on the way of reaching $T_{\mathrm{x}}$ (Scudino et al 2009). Therefore, $T_{\mathrm{x}}$ has a great impact on the liquid behaviour. Myung et al (2001) have also shown the importance of $T_{\mathrm{x}}$ in a study of viscous flow behaviour of glassy alloy with temperature. In the present study, crystallization onset temperature, $T_{\mathrm{x}}$, is selected as the third point. Hence, three sets of data points are $\left(\eta_{1}, T_{1}\right),\left(\eta_{\mathrm{x}}, T_{\mathrm{x}}\right)$ and $\left(\eta_{\mathrm{g}}, T_{\mathrm{g}}\right)$. The experimental data are taken from several literatures and also cited in tables 1-3. In most of the cases, data are not available exactly on the mentioned points. Therefore, the data nearest to these junction points are taken. The resultant function of (1) after the solution by feeding three data sets is obtained. Literatures are available where the experimental viscosity data have been fitted according to VFT equation (e.g. Mukherjee et al 2004) or any other empirical equations (Cohen and Grest 1979; Avramov 2011) and the equation parameters are reported by the respective authors. The equation parameters obtained in the available literatures by different investigators are mentioned as 'best-fit' in this paper. TAE and TSE are evaluated on the basis of the experimental data available in the literature for the present study after obtaining the analytical solution by three data sets. Moreover, TAE and TSE values are also calculated for the 'best-fits' (existing fits for the available data points in the literature) and 


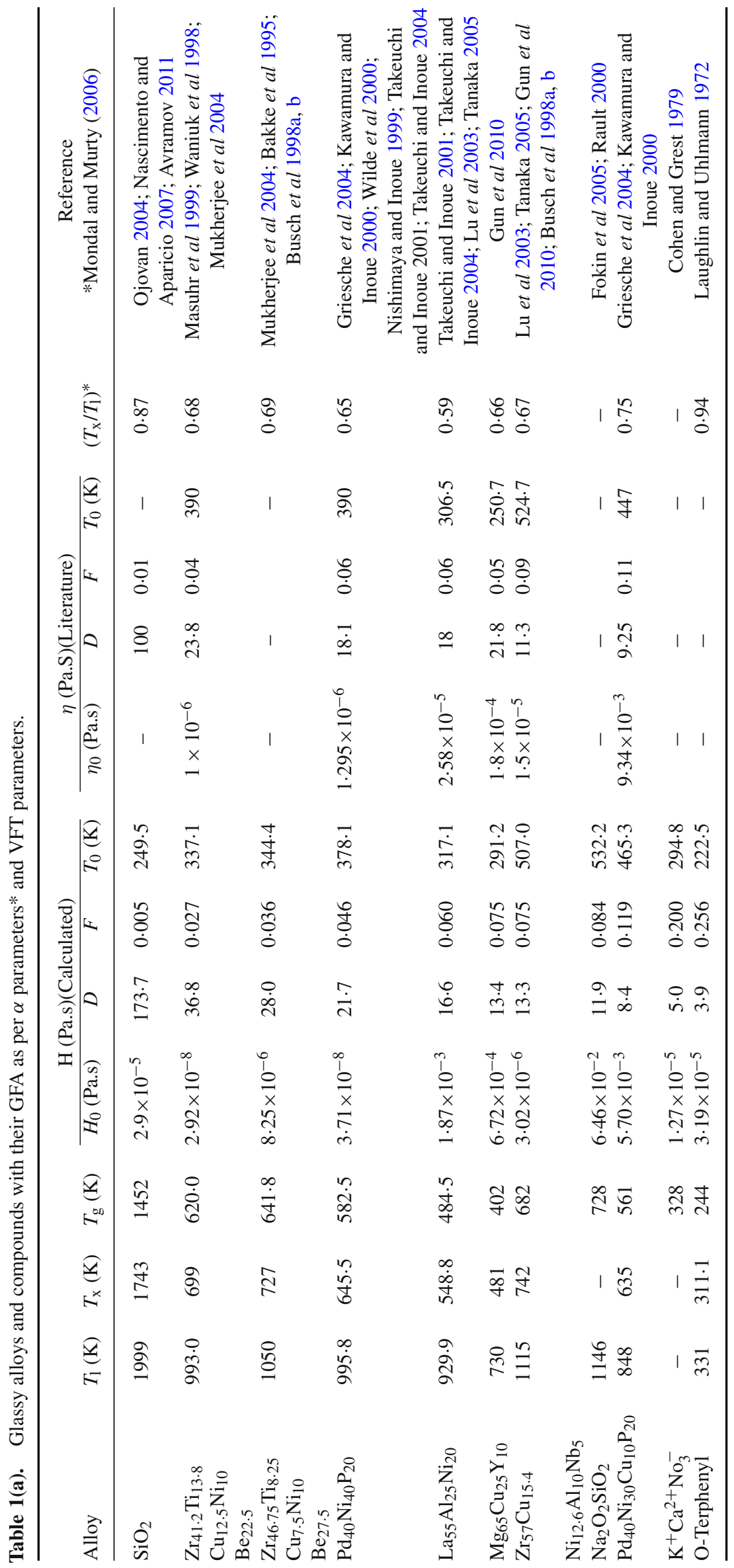




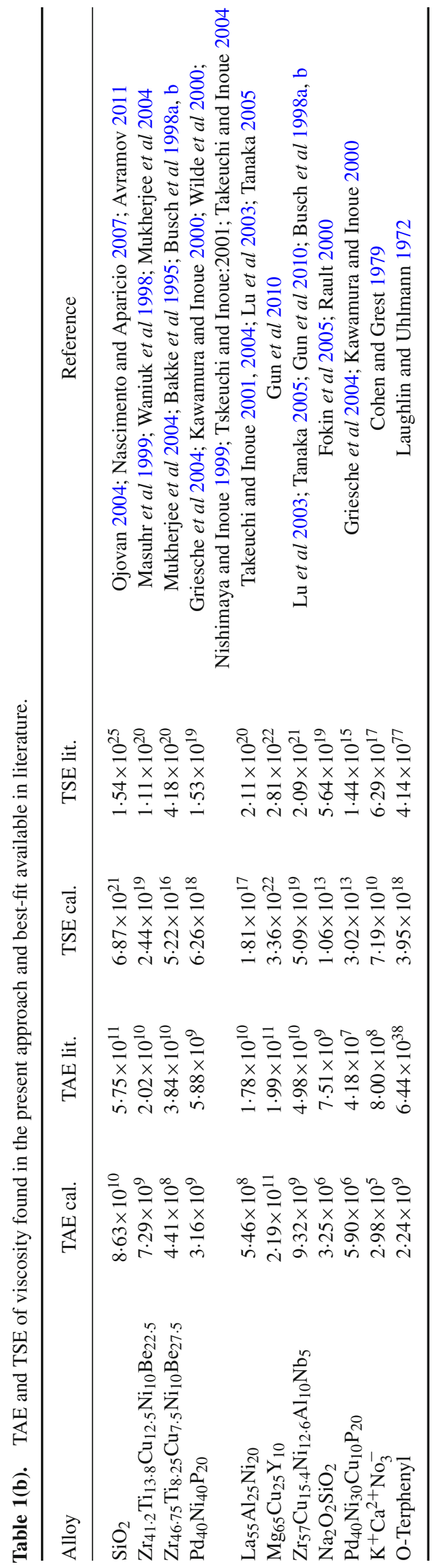




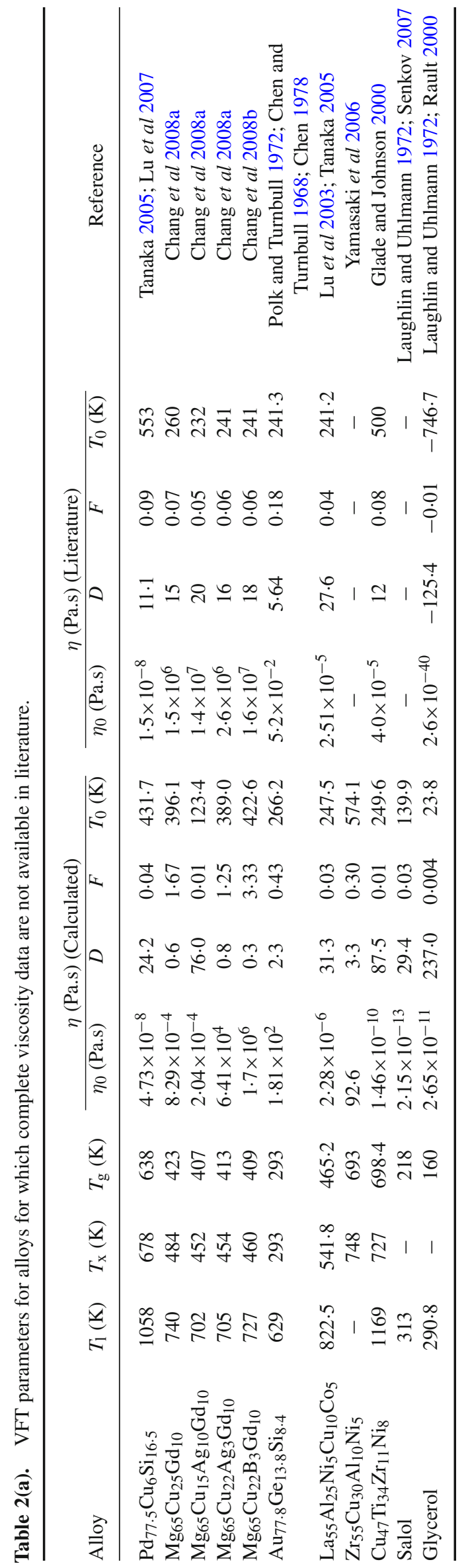




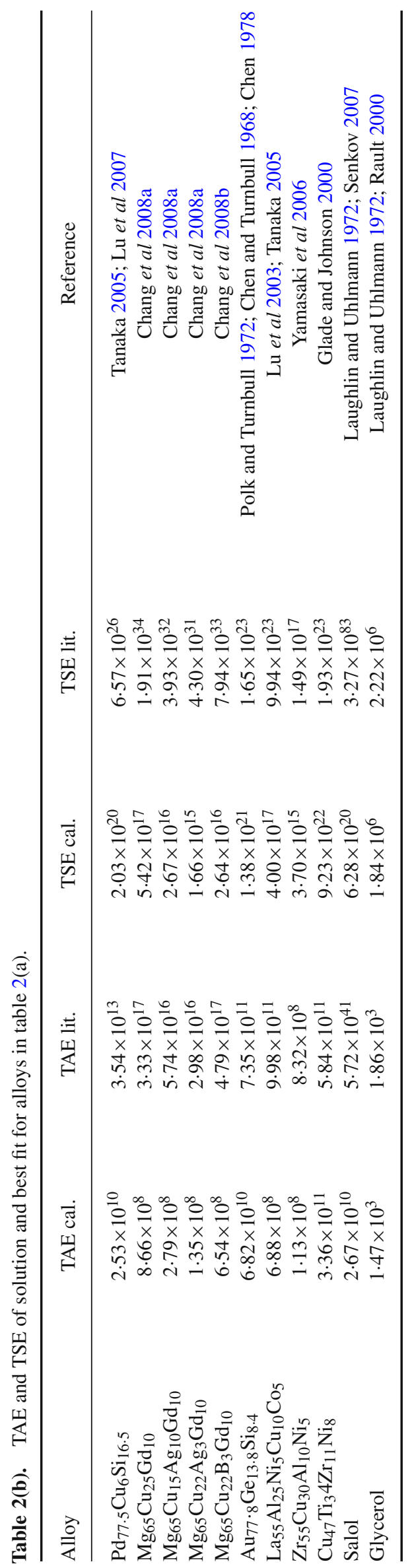


Table 3(a). 15 experimental viscosity data for alloy Vit 1.

Serial No

\begin{tabular}{lcc}
\hline 1 & $993\left(T_{1}\right)$ & $4 \cdot 835$ \\
2 & $979 \cdot 4629$ & $6 \cdot 531306$ \\
3 & $964 \cdot 2302$ & $11 \cdot 04079$ \\
4 & $948 \cdot 0122$ & $16 \cdot 36817$ \\
5 & $935 \cdot 1433$ & $24 \cdot 21029$ \\
6 & $918 \cdot 5185$ & $40 \cdot 92607$ \\
7 & $699 \cdot 7743\left(T_{\mathrm{x}}\right)$ & 17741895 \\
8 & $690 \cdot 4232$ & 44463127 \\
9 & $680 \cdot 5708$ & $1.45 \times 10^{8}$ \\
10 & $670 \cdot 2703$ & $4 \cdot 71 \times 10^{8}$ \\
11 & 660.9808 & $1 \cdot 53 \times 10^{9}$ \\
12 & $648 \cdot 5356$ & $4.99 \times 10^{9}$ \\
13 & $640 \cdot 4959$ & $1 \cdot 85 \times 10^{10}$ \\
14 & $630 \cdot 0813$ & $6 \cdot 85 \times 10^{10}$ \\
15 & $620\left(T_{\mathrm{g}}\right)$ & $3 \cdot 31 \times 10^{11}$ \\
\hline
\end{tabular}
viscosity (Pa.s)

Experimentally measured

Table 3(b). Different sets of data from table 3(a) with their corresponding solutions and TAE and TSE.

\begin{tabular}{|c|c|c|c|c|c|c|}
\hline Data set & $\eta_{0}(\mathrm{~Pa} . \mathrm{S})$ & $D$ & $F$ & $T_{0}$ & TAE & TSE \\
\hline$(1,7,15)$ & $7 \cdot 88 \times 10^{-8}$ & $32 \cdot 67$ & 0.031 & 351.91 & $5 \cdot 10 \times 10^{9}$ & $1.00 \times 10^{19}$ \\
\hline$(1,6,15)$ & $1 \cdot 50 \times 10^{-6}$ & 22.59 & 0.044 & 395.97 & $1 \cdot 8610^{10}$ & $1.34 \times 10^{20}$ \\
\hline$(1,8,15)$ & $7 \cdot 64 \times 10^{-8}$ & 32.79 & $0 \cdot 030$ & 351.45 & $5 \cdot 17 \times 10^{9}$ & $1.01 \times 10^{19}$ \\
\hline$(1,5,15)$ & $1.43 \times 10^{-6}$ & 22.73 & $0 \cdot 044$ & $395 \cdot 28$ & $1.83 \times 10^{9}$ & $1.30 \times 10^{20}$ \\
\hline$(1,9,15)$ & $2 \cdot 86 \times 10^{-8}$ & $36 \cdot 92$ & 0.027 & $336 \cdot 75$ & $7 \cdot 35 \times 10^{9}$ & $2.52 \times 10^{19}$ \\
\hline$(1,7,14)$ & $7 \cdot 39 \times 10^{-8}$ & 32.97 & 0.030 & $350 \cdot 65$ & $1 \cdot 30 \times 10^{10}$ & $8.61 \times 10^{19}$ \\
\hline$(1,7,13)$ & $1.46 \times 10^{-7}$ & 29.94 & 0.033 & $363 \cdot 86$ & $1.21 \times 10^{11}$ & $1.06 \times 10^{22}$ \\
\hline$(2,7,15)$ & $5 \cdot 62 \times 10^{-8}$ & $33 \cdot 52$ & $0 \cdot 030$ & $349 \cdot 17$ & $5 \cdot 26 \times 10^{9}$ & $1.03 \times 10^{19}$ \\
\hline$(3,7,15)$ & $7 \cdot 35 \times 10^{-8}$ & $32 \cdot 84$ & $0 \cdot 030$ & $351 \cdot 35$ & $5 \cdot 13 \times 10^{9}$ & $1.01 \times 10^{19}$ \\
\hline$(2,7,14)$ & $5 \cdot 19 \times 10^{-8}$ & $33 \cdot 89$ & $0 \cdot 030$ & 3473.68 & $1 \cdot 38 \times 10^{10}$ & $1.10 \times 10^{20}$ \\
\hline
\end{tabular}

are compared with those obtained in present approach. If at a temperature, $T_{i}$, the experimental viscosity, viscosity according to present approach and the calculated viscosity according to any given literature fitting (best-fit) are $\eta_{\text {exp }}^{i}, \eta_{\text {cal }}^{i}$ and $\eta_{\text {lit }}^{i}$, respectively then TAE and TSE for the present work are

$$
\mathrm{TAE}_{\mathrm{c}}=\sum_{i=1}^{n}\left|\eta_{\mathrm{exp}}^{i}-\eta_{\mathrm{cal}}^{i}\right|
$$

and

$$
\mathrm{TSE}_{\mathrm{c}}=\sum_{i=1}^{n}\left(\eta_{\mathrm{exp}}^{i}-\eta_{\mathrm{cal}}^{i}\right)^{2} .
$$

Similarly, TAE and TSE for the best-fit available in the literature are

$$
\mathrm{TAE}_{\mathrm{l}}=\sum_{i=1}^{n}\left|\eta_{\mathrm{exp}}^{i}-\eta_{\text {lit }}^{i}\right|
$$

and

$$
\mathrm{TSE}_{\mathrm{l}}=\sum_{i=1}^{n}\left(\eta_{\mathrm{exp}}^{i}-\eta_{\text {lit }}^{i}\right)^{2} .
$$

It has been found that several authors have not fitted the experimental viscosity data according to VFT equation (Cohen and Grest 1979; Avramov 2011) rather, they have taken the functional form of viscosity in some different way. It has also been found that in some research work (Laughlin and Uhlmann 1972; Mukherjee et al 2004) viscosity data are considered in the temperature range from temperature higher than $T_{1}$ to much lower than $T_{\mathrm{g}}$ and these data have been fitted as per VFT equation (Laughlin and Uhlmann 1972; Mukherjee et al 2004). Avramov (2011) reduced the numbers of constant parameters of VFT equation from three to two by assuming that viscosity takes a constant value of $10^{13.5} \mathrm{~Pa} . \mathrm{s}$ at $T_{\mathrm{g}}$. Thus, VFT equation converts to

$$
\log \eta=\log \eta_{v}+\left(13 \cdot 5-\log \eta_{v}\right)\left\{\left(T_{\mathrm{g}}-T_{0}\right) /\left(T-T_{0}\right)\right\},
$$


where, $\eta_{v}$ is a pre-exponential constant and the experimental viscosity data for $\mathrm{SiO}_{2}$ are fitted according to this equation. Cohen and Grest (1979) have extended the free volume approach and proposed a temperature dependence of viscosity for ionic liquids like $\mathrm{K}^{+} \mathrm{Ca}^{2+} \mathrm{NO}_{3}^{-}$as follows:

$$
\log _{10} \eta=A+2 B /\left[T-T_{0}+\left\{\left(T-T_{0}\right)^{2}+4 v_{\mathrm{a}} \zeta_{0} T\right\}^{1 / 2}\right]
$$

where, $B=v_{m} \zeta_{0} \log _{10} e$,

where $v_{\mathrm{a}}$ is a constant having dimension of volume, $\zeta_{0}$ a constant and $v_{\mathrm{m}}$ the molecular volume (Cohen and Grest 1979). However, VFT equation does not hold good beyond the temperature range $T_{1}$ to $T_{\mathrm{g}}$ (Battezzati and Greer 1989). Thus, experimental viscosities in between $T_{1}$ and $T_{\mathrm{g}}$ (excluding the data beyond this range) are considered here. Table 1(a) shows a number of alloys and compounds where glass formation is reported (see 'Reference' column of table 1(a)) and the present method of estimation of viscosity is applied to all these systems.

Several reports of viscosity data are available (reference column of table 2(a)) where the data are reported for a very short range of temperature, e.g. from near to $T_{\mathrm{x}}$ to $T_{\mathrm{g}}$. Still, the present method is applied to calculate the analytical solution of VFT equation in the given temperature range taking two points at two extremities of the reported temperature range and the third one is chosen in the middle of the two and the analytical solution is obtained and compared to the reported best-fit values too. However, as the data of the entire temperature range is not available, the solution obtained serves basically as an accurate way than the bestfits reported in literature to interpolate viscosity values in between the given temperature range only. Thus, values of the fragility parameter obtained in the present approach utilizing these incomplete data (as it is not available over the entire range from $T_{1}$ to $T_{\mathrm{g}}$ ) cannot be expected to reflect the exact glassy behaviour of the alloy. The reason is solution of (1) greatly depends on three data points $\left(T_{\mathrm{l}}, T_{\mathrm{x}}\right.$ and $\left.T_{\mathrm{g}}\right)$ and altering any of three points to such an extent has a large impact on the solution. Hence, it proves the necessity of the data at three junction temperatures $\left(T_{\mathrm{l}}, T_{\mathrm{x}}\right.$ and $\left.T_{\mathrm{g}}\right)$.

In order to check appropriateness of the choice of viscosity data at three critical temperatures $\left(T_{1}, T_{\mathrm{x}}\right.$ and $\left.T_{\mathrm{g}}\right)$, one of the three data (temperature-viscosity) points is systematically altered while keeping other two data points unchanged. Similarly, two other data points are changed while keeping only one of the three unchanged. For each and every set of three data points, a different solution (of equation 1) is achieved with three constant viscosity parameters $\left(\eta_{0}, D\right.$ and $\left.T_{0}\right)$. TAE and TSE have been calculated for each and every solution set with respect to the experimental data points available in the entire range of interest ( $T_{1}$ to $T_{\mathrm{g}}$ ). For example, 15 numbers of experimentally determined temperature-viscosity data for Vit 1 are shown by solid square in figure 1 (Mukherjee et al 2004). Serial numbers are assigned to all the data points (figure 1). The numerical values of the data are given later in table 3(a). Table 3(b) shows different combinations of

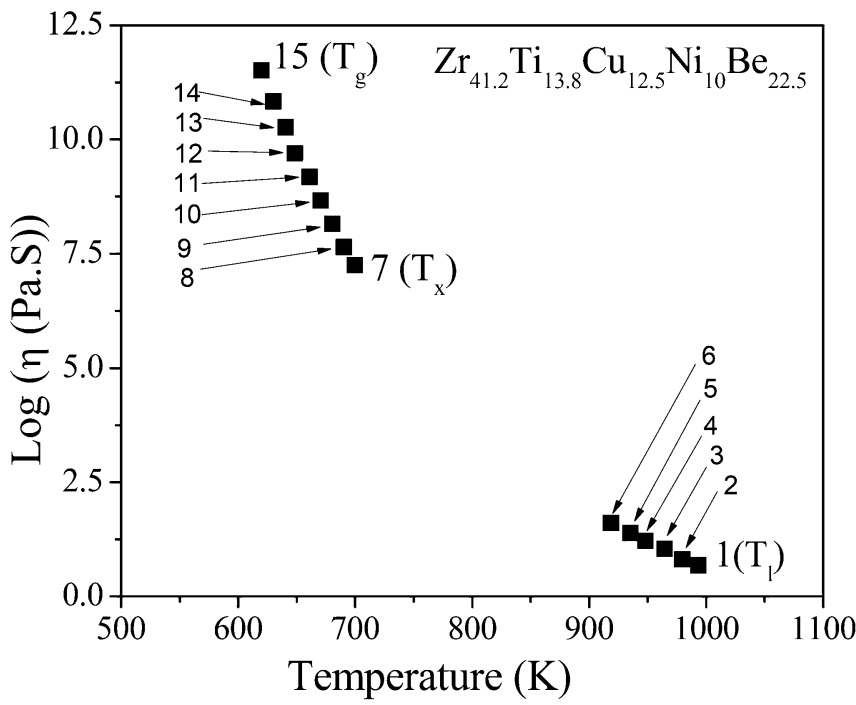

Figure 1. 15 experimental data points with each of points numbered for Vit 1 (Mukherjee et al 2004).

different data points as per the above process $\{$ e.g. $(1,7,15)$, then the midpoint is shifted $(1,6,15)$, later midpoint is kept fixed and the extremities are shifted $(2,7,14)\}$.

\section{Results and discussion}

Viscosity data from the $T_{1}$ to $T_{\mathrm{g}}$ are available for all the alloys and compounds mentioned in table $1(\mathrm{a}) . T_{1}, T_{\mathrm{x}}$ and $T_{\mathrm{g}}$ are cited for each material in table 1(a). VFT parameters calculated by the present method and as reported in the literature considered as the 'best-fit' are also shown. In those cases where VFT parameters are not available since viscosity fitting is not in the VFT mode in the literature (Cohen and Grest 1979; Avramov 2011), the values of $\eta_{0}, D$ and $T_{0}$ are not shown. If viscosities at the mentioned temperatures are not available, temperature and viscosity nearest to that 'junction temperature' are considered. The alloys are arranged as per the decreasing $D$ values according to the solution obtained by the present method. Fragility $(F)$ values of the glassy materials as per Angel (1986) are also added in table 1(a). The arrangement according to the $D$ values matches very well with decreasing GFAs of the alloys. The values of fragility parameters obtained from 'best-fits' of literature do not match so well, e.g. Vit 1 and $\mathrm{Mg}_{65} \mathrm{Cu}_{25} \mathrm{Y}_{10}$ have been found to have comparable fragilities (23.8 and 21.8, respectively) according to literature (Mukherjee et al 2004; Gun et al 2010). However, in reality, the former is considered to be far better glass former (much higher cast section thickness) and it is significantly revealed in the present approach (table 1(a)) since it has much higher $D$ value compared to the later alloy. Moreover, the best known glass former so far is $\mathrm{SiO}_{2}$ for which the $D$ value is found to be 100 (Nascimento and Aparicio 2007), but the present approach shows much better GFA of $\mathrm{SiO}_{2}$ because of its very high $D$ value (173.7). 
A widely used GFA criterion, $\alpha$-parameter $\left(T_{\mathrm{x}} / T_{1}\right)$, from the work of Mondal et al (Mondal and Murty 2006) is considered to compare competence of the present approach for predicting GFA of glassy alloys as its added advantage. The criteria explains GFA of the alloys like $\mathrm{SiO}_{2}$ and Vit 1 and others with high GFA, however, it fails for very fragile organic compounds like O-terphenyl. However, the present approach is able to reveal its poor GFA from its very low $D$ value (=3.9). Moreover, according to the $\alpha$-parameter (Mondal and Murty 2006), $\mathrm{Mg}_{65} \mathrm{Cu}_{25} \mathrm{Y}_{10}$ shows a $T_{\mathrm{x}} / T_{1}$ ratio of 0.66 which is in the range of strong glass formers like Vit 1. However, in experimental conditions, it has been experienced that the alloy can be cast around $4 \mathrm{~mm}$ in diameter (Lu et al 2007) which invariably speaks about its poorer GFA compared to Vit 1 and this is clear from its very low $D$ value obtained in the present approach. Table 1(b) shows TAE and TSE of the present approach and the best-fit in the literature with respect to the available experimental viscosity data points. For each and every alloy, TAE values of the present study are observed to have at least one order of magnitude less than those of the best-fit in the literature.

Figure 2( $a$ and $b$ ) shows plots of logarithm of viscosity as a function of inverse of temperature obtained by experimental route (as experimental (indents)) (Laughlin and Uhlmann 1972; Ojovan 2004; Nascimento and Aparicio 2007; Avramov 2011, present method (solid line with hollow triangle indents)), best-fit available in literature (dashed line with hollow circular indents)) for $\mathrm{SiO}_{2}$ (a very strong liquid) and O-terphenyl (a very fragile liquid), respectively. Careful investigation of figure 2 shows that in case of $\mathrm{SiO}_{2}$ (figure 2a) the solution of present method (calculated) lies exactly on every experimental data points making it far better than the best-fit of the literature, which is far away than the experimental data in the entire temperature range from $T_{1}$ to $T_{\mathrm{g}}$. Furthermore, figure 2(b) elaborates the accuracy of forecasting of the solution of present method, and the bestfit of the literature is better in the higher temperature range. However, as the temperature decreases, the solution of bestfit shoots up to a very high value of viscosity even more than 6 orders of magnitude than the corresponding experimental data resulting in a huge error of the best-fit in TAE and TSE.
Table 2(a) is a list of alloys with their experimental viscosity data in the temperature range between $T_{\mathrm{x}}$ and $T_{\mathrm{g}}$. Table 2(a) shows VFT parameters obtained in the present approach for a number of glassy alloys and compounds where complete viscosity data are not available in the literature. Fragility $(F)$ values of the glassy materials as per Angel (1986) are also shown in table 2(a). The solution in table 2(a) is obtained as per (2-4) by considering viscosities at the uppermost and lower most temperatures and the third point is the middle in the temperature range. The solution shows several orders of magnitude less errors with respect to the experimental data points as compared to the best-fit reported in the literature (table 2(b)). The solution obtained here does not involve one nodal point $\left(T_{1}\right)$ of the three needed to achieve a solution that can reflect the actual glassy behaviour of the alloy. Thus, the solution in this case can well be applied as a better interpolation tool to acquire viscosity data at any temperature within the given range. However, the solution can not be used for forecasting GFA of any alloy. According to table 2(a), the $D$ values of organic liquids like salol or glycerol are as high as 29.4 and 237.0 which are even larger than one of the best glass formers found so far, $\mathrm{SiO}_{2}$ (table 1(a)). This is understandable that in case of polymeric materials, viscosity is decided by the molecular weight and it is difficult to understand fragility without proper modification (Angel 1995). A very poor glass former, $\mathrm{Cu}_{47} \mathrm{Ti}_{34} \mathrm{Zr}_{11} \mathrm{Ni}_{8}$, with respect to their actual behaviours (Tanaka 2005; Lu et al 2007; Glade and Johnson 2000) shows a $D$ value (87.5) much larger than $\mathrm{Pd}_{77.5} \mathrm{Cu}_{6} \mathrm{Si}_{16.5}$ which is highly untenable with respect to its actual behaviour.

Table 3(a) shows experimental viscosity data over the entire temperature range between $T_{1}$ and $T_{\mathrm{g}}$ for the alloy Vit 1 (figure 1) (Mukherjee et al 2004). Table 3(b) shows systematic alteration of data set and their resultant VFT parameters along with TAE and TSE with respect to the experimentally observed values for each data set. It is interesting to note observation number 1 i.e. the solution with $T_{1}, T_{\mathrm{x}}$ and $T_{\mathrm{g}}$ containing the least error among all. The error and the VFT parameters undergo a very minute change if the data point is shifted down from $T_{1}$ keeping the other two points on $T_{\mathrm{x}}$ and $T_{\mathrm{g}}$ fixed. However, if a point is shifted up from $T_{\mathrm{g}}$
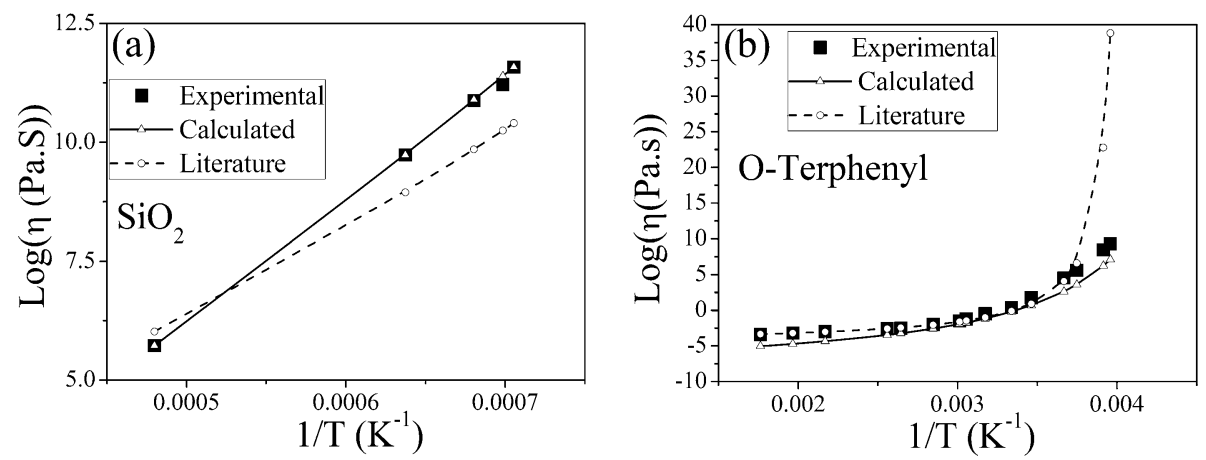

Figure 2. Plots of logarithm of viscosity vs $1 / T$ of (a) one of best glass former, $\mathrm{SiO}_{2}$ and (b) one of worst glass former, O-terphenyl. 
keeping other two points at $T_{1}$ and $T_{\mathrm{x}}$ fixed, the solution and the error face a considerable change where TAE increases one to three orders of magnitude. The basic reason for the better result in the present method of solution is attributed to the choice of data points. The fitting method adopted here tries to minimize the error since the present method takes care of three main junction points. It is already said that $T_{1}$ being a crystal to solid first order phase transition temperature, $T_{\mathrm{x}}$ being the transition temperature of glass to crystal upon heating and $T_{\mathrm{g}}$ being the kinetic glass transition temperature are surely the junction temperatures and they are very critical with respect to glass transition and viscosity variation. Furthermore, it is very interesting to note that if $T_{1}$ and $T_{\mathrm{g}}$ are kept constant, and $T_{\mathrm{x}}$ varies, the resultant solution varies slightly and especially, the $D$ value faces a very minute change if $T_{\mathrm{x}}$ increases. Generally, the critical temperatures are evaluated with slowest possible scan rate in differential scanning calorimetry (DSC) and as the heating rate increases, $T_{\mathrm{x}}$ also increases. Resultantly, the solution and the $D$ values do not face a considerable change.

However, in spite of being excellent glass formers, Pdbased alloys have shown very low $D$ value (table 1(a)). Fan et al (2004) have shown that although Pd-based bulk metallic glasses are very fragile liquid, still they show good GFA because only kinetic factor such as viscosity is not sufficient to explain GFA of Pd-based BMGs. They (Fan et al 2004) have further shown that there exists smaller thermodynamic driving force and larger diffusion length for crystal nucleation and growth for these alloys. Thus, Pd-based BMGs behave somewhat anomalously in glass formability with respect to its $D$ values.

The principal aims of the present approach is to achieve a solution for viscosity as a function of temperature where the solution will contain least possible error along with VFT parameters reflecting the real behaviour of the liquid, thus, giving a forecast of its GFA at a glance. As the VFT equation contains an exponential function, the number and quality of data has very significant importance. In table 1(a and b), TAE and TSE are less in case of present method which can be explained as in most of the cases, the experimental viscosity data have been taken from well above the $T_{1}$ to well below the $T_{\mathrm{g}}$. However, in the present paper, only $T_{1}$ to $T_{\mathrm{g}}$ range is considered and the solution and the 'best-fit' are compared with respect to those many experimental data points which fall inside the mentioned range. Moreover, the way of fitting has not been reported in the literature. The fitting method utilized for the alloys and compounds in table $1(\mathrm{a}$ and $\mathrm{b})$ is greatly contributed by those data points outside the range of $T_{1}$ to $T_{\mathrm{g}}$. The 'best-fit' may prove to be better if all the experimental points outside the range are considered. However, it is mentioned well before that VFT equation does not hold well beyond the temperature range except $T_{1}$ to $T_{\mathrm{g}}$. For the alloys listed in table 2(a and b), TAE and TSE are still lower for the present method. There may be some very small error in taking the data from the graph and the values of viscosity in that temperature range are very high (in the order of $10^{5}-10^{12}$ ), even a slight error in data is capable of creating huge error with respect to 'best-fit'. It is of prime importance to show in the present work that there is no necessity of determining a huge number of experimental viscosity values to determine its viscosity behaviour in the temperature range between $T_{1}$ and $T_{\mathrm{g}}$. Only three viscosity data at three mentioned temperatures or as near as possible to those are required to achieve an analytical solution of VFT parameters that can satisfactorily explain the viscosity behaviour of a glassy alloy or compound in the temperature range $T_{1}$ to $T_{\mathrm{g}}$. The present method is at least comparable to the 'best-fit' method and often much better as seen in the cases investigated in this work. Moreover, the experimental errors increase as the number of data points to be taken increases and that invariably increases the chance to incorporate errors in the 'best-fit'. It is interesting to note that the present method serves as an extra advantage with its modified fragility values that satisfactorily forecasts GFA of some of the glassy alloys and compounds. Table 3(b) is an excellent example of extreme importance of choice of data points. Special care is to be given while evaluating $T_{1}, T_{\mathrm{x}}$ and $T_{\mathrm{g}}$ of any alloy. As in table 3(b), it is evident that a little deviation would not only increase the error in solution but the resultant VFT parameters may show some erroneous values. $T_{\mathrm{x}}$ temperature is to be evaluated via lowest possible heating rate in DSC because, increase in $T_{\mathrm{x}}$ does not affect the solution that much as the decrease in $T_{\mathrm{x}}$. Thus, the obtained $T_{\mathrm{x}}$ must be as lowest as possible.

\section{Conclusions}

A method based on analytical solution for evaluating viscosity as a function of temperature is established where the temperature range of interest is $T_{1}$ to $T_{\mathrm{g}}$. Viscosity data at $T_{1}, T_{\mathrm{x}}$ and $T_{\mathrm{g}}$ are most important and they are used to achieve the analytical solution of VFT equation to estimate VFT parameters. The experimental data set for which the solution has been reported in literature is used to achieve analytical solution and both the methods are compared with respect to the experimental data set. The solution of present method is found to have at least one order of magnitude less error (TAE) than the 'best-fit' of literature. The method has also been applied where the viscosity data are not available for the mentioned temperature range. Still, the analytical solution shows better interpolation than the 'best-fit' reported in literature. The idea of more the number of experimental data, the better is the solution that has been removed in this method. Only three optimally chosen data points serve the purpose with several other favourable outcomes.

Most importantly, VFT parameters obtained through the analytical solution method when applied to the data with complete range of temperature $\left(T_{1}\right.$ to $\left.T_{\mathrm{g}}\right)$, can prove to be a reliable testimonial for GFA. The fragility parameter $D$ obtained through this analytical solution method is observed to explain the GFAs of almost all kinds of glasses starting from inorganic alloys to ionic and organic liquids. The main objective to find out viscosity fitting with undercooling of 
glassy liquids has successfully been accomplished. The GFA part is an interesting outcome of the present work.

\section{References}

Angel C A 1986 Ann. N. Y. Acad. Sci 486241

Angel C A 1988 J. Phys. Chem. Solids 49863

Angel C A 1995 Science New Series 2671924

Avramov I 2011 J. Non-Cryst. Solids 357391

Bakke E, Busch R and Johnson W L 1995 Appl. Phys. Lett. 673260

Battezzati L and Greer A L 1989 Acta Metall. 371791

Busch R 2000 JOM 5239

Busch R, Bakke E and Johnson W L 1998a Acta Mater. 464725

Busch R, Liu W and Johnson W L 1998b J. Appl. Phys. 834134

Chang Y C, Huang J C, Cheng Y T, Lee C J, Du X H and Nieh T G 2008a J. Appl. Phys. 103103521

Chang Y C, Huang J C, Tang C W, Chang C I and Jang J S C 2008b Mater. Trans. (JIM) 492605

Chen H S 1978 J. Non-Cryst. Solids 27257

Chen H S and Turnbull D 1968 J. Chem. Phys. 482560

Cohen M H and Grest G S 1979 Phys. Rev. B20 1077

Doolittle A K 1951 J. Appl. Phys. 221471

Fan G J, Fecht H J and Lavernia E J 2004 Appl. Phys. Lett. 84487

Fokin V M, Nascimento M L F and Zanotto E D 2005 J. Non-Cryst. Solid $\mathbf{3 5 1} 789$

Glade S C and Johnson W L 2000 J. Appl. Phys. 877249

Griesche A, Macht M P, Garandet J P and Frohberg G 2004 J. NonCryst. Solid 336173

Gun B, Laws K J and Ferry M 2010 J. Alloys Compds. 496582
Hong H H, Li Y, Ng S C and Ong C K 1996 J. Non-Cryst. Solids 208127

Kawamura Y and Inoue A 2000 Appl. Phys. Lett. 771114

Laughlin W T and Uhlmann D R 1972 J. Phys. Chem. 782317

Lu Z P, Li Y and Liu C T 2003 J. Appl. Phys. 93286

Lu Z P, Bei H and Liu C T 2007 Intermetallics 15618

Masuhr A, Waniuk T A, Busch R and Johnson W L 1999 Phys. Rev. Lett. 822290

Mondal K and Murty B S 2006 J. Non-Cryst. Solid 3525257

Mukherjee S, Schroers J, Zhou Z, Johnson W L and Rhim W K 2004 Acta Mater. 523689

Myung W N, Ryu S P, Hwang I S, Kimb H G, Zhangc T, Inoue A and Greer A L 2001 Mater. Sci. Eng. A304-306 691

Nascimento M L F and Aparicio C 2007 Physica B398 71

Nishimaya N and Inoue A 1999 Acta Mater. 471487

Ojovan M I 2004 JETP Lett. 79632

Polk D E and Turnbull D 1972 Acta Metal. 20493

Rault J 2000 J. Non-Cryst. Solids 271177

Rao K J, Bhatt M H and Kumar S 2001 J. Indian Inst. Sci. 81 3-13

Scudino S, Bartusch B and Eckert J 2009 J. Phys. Conf. Ser. 144 012097

Senkov O N 2007 Phys. Rev. B76 104202

Takeuchi A and Inoue A 2001 Mater. Sci. Engg. A304-306 446

Takeuchi A and Inoue A 2004 Mater. Sci. Engg. A375-377 449

Wilde G, Gorler G P and Willnecker R 2000 J. Appl. Phys. 871141

Waniuk T A, Bush R, Mashur A and Johnson W L 1998 Acta Mater. 465229

Tanaka H 2005 J. Non-Cryst. Solids 351678

Yamasaki T, Maeda S, Yokoyama Y, Okai D, Fukami T, Kimura H $\mathrm{M}$ and Inoue A 2006 Intermetallics 141102 